\title{
Do schizotypal or borderline personality disorders predict onset of psychotic disorder or persistent attenuated psychotic symptoms in patients at high clinical risk?
}

The prevalence and predictive role of personality disorders in ultra-high risk (UHR) for psychosis patients has been a comparatively neglected area of focus in predictive modelling research in this field. Examining the predictive significance of personality disorders in this clinical population is particularly indicated given the phenomenological overlap of some personalty disorders, most notably schizotypal personality disorder (SPD) and borderline personality disorder (BPD), with psychosis. In fact, a recent study found that the majority of a sample of BPD patients ( $70 \%)$ met the criteria for a schizophrenia spectrum disorder (Zandersen \& Parnas, 2019). Indeed, there has been recent discussion that the BPD diagnosis is over-inclusive and clinically and conceptually impossible to distinguish from schizophrenia spectrum disorders (Zandersen et al., 2019). SPD includes a range of psychosis-related disturbances, such as ideas of reference, odd beliefs, unusual perceptual experiences, odd thinking/speech, paranoid ideaetion, inappropriate/constricted affect, and odd behaviour/appearance, as part of its defining criteria in DSM 5.

Due to the phenomenological overlap of BPD and SPD with psychotic disorders and their reasonably high prevalence in UHR patients in previous analyses (Ruhrmann et al., 2010; Ryan et al., 2017; Salokangas et al., 2013; Schmidt et al., 2017; Schultze-Lutter et al., 2012; Schroeder et al., 2013), we preformed a secondary analysis of the Neurapro trial of omega-3 fatty acids in UHR young people (see Markulev et al, 2017; McGorry et al., 2017). Our aims were to examine whether these personality disorders would be more prevalent in a UHR sample than in the general population (estimated to be $1.7 \%$ for BPD and $4 \%$ for SPD); and whether UHR patients with either SPD or BPD would have higher rates of transition to psychosis and more persistent attenuated psychotic symptoms, compared to UHR patients without these personality disorders.

Three hundred and four UHR patients aged 13 and 39 years (165 [54\%] females, $M$ age = $19.12, S D=4.55)$ were recruited from ten early psychosis clinics internationally. Particpants were assessed for transition to psychosis (CAARMS), presence of SPD and BPD (SCID-II), persistent attenuated psychotic symptoms (meeting the UHR attenuated psychotic symptoms 
risk criteria at baseline and at 12-month follow up), general psychopathology (BPRS), and severity of attenuated psychotic symptoms (CAARMS positive symptom subscale). Results showed that of the 293 participants who had baseline data available on personality disorders, 19 cases met criteria for BPD (6.5\%) and 9 cases (3\%) for SPD, corresponding to $9.55 \%$ of the sample meeting criteria for one of these personality disorders (no participants met criteria for both). For baseline demographics and clinical characteristics see Table S1. A total of 40 cases were known to have transitioned to psychosis. Of those who were diagnosed with SPD or BPD at baseline, $44 \%$ and $11 \%$ had transitioned to psychosis, respectively (Table S2). The presence of SPD was a significant predictor of transition, and remained so after adjusting for recruitment site and age (as they varied with SPD status) and general psychopathology (Table S3; Figure S1). Conversely, the presence of a BPD diagnosis did not predict transition to psychosis $(p=.93)$, with a hazard ratio of 1.07 for BPD vs. non-BPD, 95\% CI: [0.26, 4.43] (Table S3, Figure S2). The presence of a personality disorder diagnosis (SPD or BPD) did not predict persistent attenuated psychotic symptoms at 12-month follow-up in the nontransitioned cases $\left(R^{2}=0.023,95 \%\right.$ CI $\left.[0.00,0.065], F(2,190)=2.22, p=.112\right)$. Neither $\operatorname{SPD}\left(b=-0.69,95 \%\right.$ CI $[0.031,8.20]$, Wald $\left.\chi^{2}(1)=0.23, p=.640\right)$ nor BPD $(b=-0.91,95 \%$ CI $[0.105,1.56]$, Wald $\left.\chi^{2}(1)=1.74, p=.187\right)$ were significant predictors of attenuated psychotic symptom persistence at follow-up.

In line with our expectations, the prevalence of BPD was higher (6.5\%) in our UHR sample compared to the general population. However, in contrast to previous reports (Rosen et al., 2006; Schultze-Lutter et al., 2012; Sevilla-Llewellyn-Jones et al., 2018), the prevalence of SPD was similar (3\%) to that of the general population. SPD but not BPD predicted transition to psychosis over the follow up period. The lack of predictive value of BPD for onset of psychotic disorder is consistent with previous reports from our group (Ryan et al., 2017; Thompson et al., 2012).

Schizotypal personality features and threshold SPD, however, have been found to have predictive value in previous studies (Ruhrmann et al., 2010; Schmidt et al., 2017), consistent with the current findings. SPD may be a distinct marker of psychosis risk among UHR individuals, supported by the fact it emerged as a significant predictor even after adjusting for other relevant variables (general psychopathology, recruitment site, age). BPD, on the other hand, despite its phenomenological overlap with schizophrenia spectrum disorders, may not 
be. However, the reasonably low prevalence of SPD in UHR samples (3\% in the current sample) limits its utility as a predictive marker in UHR cohorts.

Interestingly, personality disorders did not predict persistence of attenuated psychotic symptoms in cases who did not transition to psychosis. This finding contrasts with data reported by Rutigliano et al. (2016), who found that one third of non-transitioned UHR cases with comorbid non-psychotic mental disorders experienced persistence of attenuated psychotic symptoms at 6-year follow-up. The reason for this difference is likely that the current study focused exclusively on two personality disorders, while Rutigliano et al. (2016) included both comorbid Axis I and personality disorders, with the majority of their sample (51.4\%) presenting with the former, which may have been responsible for the association with persistent attenuated psychotic symptoms.

A strength of the current study is the large sample size from international recruitment sites. However, several imitations need to be noted. Firstly, the sample size of those with personality disorders was small $(n=28)$, particularly the SPD group $(n=9)$, which limits the statistical power of the analysis and generalisability. The positive finding relating to SPD needs to be replicated with larger samples and with a larger proportion of SPD participants. Secondly, the cohort displayed a modest transition to psychosis rate (13\%), which decreased the power for testing the research question of whether these personality disorders are associated with transition risk. Finally, participants received comprehensive psychosocial intervention over the course of the study, which might have reduced the transition rate in the sample. While this is of course desirable, it might have confounded examination of the personality disorder-psychosis risk relationship.

The current study indicates increased risk of threshold-level psychotic disorder in UHR patients who present with SPD. BPD in UHR patients does not appear to be related to increased psychosis risk. This underlines the importance of a) maintaining SPD as part of the UHR criteria (in the Trait Vulnerability group) and b) screening and assessing SPD in young people accessing clinical services. Future studies could further examine the issue of the personality disorder-psychosis risk relationship by examining prevalence of UHR status and outcomes of cohorts with personality disorders. Treatment effects could be examined by including comparison groups of UHR individuals who are help-seeking yet who do not engage with treatment. 


\section{References}

1. Markulev, C., McGorry, P. D., Nelson, B., Yuen, H. P., Schaefer, M., Yung, A. R., ... Amminger, G. P. (2017). NEURAPRO-E study protocol: a multicentre randomized controlled trial of omega-3 fatty acids and cognitive-behavioural case management for patients at ultra high risk of schizophrenia and other psychotic disorders. Early Intervention in Psychiatry, 11(5), 418-428. https://doi.org/10.1111/eip.12260

2. McGorry, P. D., Nelson, B., Markulev, C., Yuen, H. P., Schäfer, M. R., Mossaheb, N., ... \& Chen, E. Y. (2017). Effect of $\omega-3$ polyunsaturated fatty acids in young people at ultrahigh risk for psychotic disorders: the NEURAPRO randomized clinical trial. JAMA psychiatry, 74(1), 19-27.

3. Newton-Howes, G., Tyrer, P., North, B., \& Yang, M. (2008). The prevalence of personality disorder in schizophrenia and psychotic disorders: Systematic review of rates and explanatory modelling. Psychological Medicine, 38(8), 1075-1082. https://doi.org/10.1017/S0033291707002036

4. Rosen, J. L., Miller, T. J., D’Andrea, J. T., Mcglashan, T. H., \& Woods, S. W. (2006). Comorbid diagnoses in patients meeting criteria for the schizophrenia prodrome. Schizophrenia Research, 85(1-3), 124-131. https://doi.org/10.1016/j.schres.2006.03.034

5. Ruhrmann, S., Schultze-Lutter, F., Salokangas, R. K. R., Heinimaa, M., Linszen, D., Dingemans, P., ... Klosterkötter, J. (2010). Prediction of psychosis in adolescents and young adults at high risk: Results from the prospective European prediction of psychosis study. Archives of General Psychiatry, 67(3), 241-251. https://doi.org/10.1001/archgenpsychiatry.2009.206

6. Rutigliano, G., Valmaggia, L., Landi, P., Frascarelli, M., Cappucciati, M., Sear, V., ... Fusar-Poli, P. (2016). Persistence or recurrence of non-psychotic comorbid mental disorders associated with 6-year poor functional outcomes in patients at ultra high risk for psychosis. Journal of Affective Disorders, 203, 101-110. https://doi.org/10.1016/j.jad.2016.05.053

7. Ryan, J., Graham, A., Nelson, B., \& Yung, A. (2017). Borderline personality pathology in young people at ultra high risk of developing a psychotic disorder. Early Intervention in Psychiatry, 11(3), 208-214. https://doi.org/10.1111/eip.12236

8. Salokangas, R. K. R., Dingemans, P., Heinimaa, M., Svirskis, T., Luutonen, S., 
Hietala, J., ... Klosterkötter, J. (2013). Prediction of psychosis in clinical high-risk patients by the Schizotypal Personality Questionnaire. Results of the EPOS project. European Psychiatry, 28(8), 469-475. https://doi.org/10.1016/j.eurpsy.2013.01.001

9. Schmidt, A., Cappucciati, M., Radua, J., Rutigliano, G., Rocchetti, M., Dell'Osso, L., ... Fusar-Poli, P. (2017). Improving Prognostic Accuracy in Subjects at Clinical High Risk for Psychosis: Systematic Review of Predictive Models and Meta-analytical Sequential Testing Simulation. Schizophrenia Bulletin, 43(2), 375-388. https://doi.org/10.1093/schbul/sbw098

10. Schroeder, K., Fisher, H. L., \& Schäfer, I. (2013). Psychotic symptoms in patients with borderline personality disorder. Current Opinion in Psychiatry, 26(1), 113-119. https://doi.org/10.1097/YCO.0b013e32835a2ae7

11. Schultze-Lutter, F., Klosterkötter, J., Michel, C., Winkler, K., \& Ruhrmann, S. (2012). Personality disorders and accentuations in at-risk persons with and without conversion to first-episode psychosis. Early Intervention in Psychiatry, 6(4), 389398. https://doi.org/10.1111/j.1751-7893.2011.00324.x

12. Sevilla-Llewellyn-Jones, J., Camino, G., Russo, D. A., Painter, M., Montejo, A. L., Ochoa, S., ... Perez, J. (2018). Clinically Significant Personality Traits in Individuals at High Risk of Developing Psychosis. Psychiatry Research. https://doi.org/10.1016/j.psychres.2018.01.027

13. Thompson, A., Nelson, B., Bechdolf, A., Chanen, A. M., Domingues, I., Mcdougall, E., \& Yung, A. R. (2012). Original Article Borderline personality features and development of psychosis in an 'Ultra High Risk' (UHR) population : a case control study. (June 2010), 1-9. https://doi.org/10.1111/j.1751-7893.2012.00365.x

14. Zandersen, M., Henriksen, M. G., \& Parnas, J. (2019). A Recurrent Question: What Is Borderline? Journal of Personality Disorders, 33(3), 341-369. https://doi.org/10.1521/pedi_2018_32_348

15. Zandersen, M., \& Parnas, J. (2019). Exploring schizophrenia spectrum psychopathology in borderline personality disorder. European Archives of Psychiatry and Clinical Neuroscience, 1-10. https://doi.org/10.1007/s00406-019-01039-4 


\section{University Library}

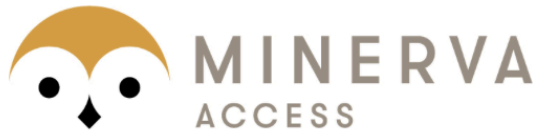

A gateway to Melbourne's research publications

Minerva Access is the Institutional Repository of The University of Melbourne

\section{Author/s:}

Hadar, H;Zhang, H;Phillips, LJ;Amminger, GP;Berger, GE;Chen, EYH;de Haan, L;Hartmann, JA;Hickie, IB;Lavoie, S;Markulev, C;McGorry, PD;Mossaheb, N;Nieman, DH;Nordentoft, M;Riecher-Rossler, A;Schafer, MR;Schloegelhofer, M;Smesny, S;Thompson, A;Verma, S;Yuen, HP;Yung, AR;Nelson, B

Title:

Do schizotypal or borderline personality disorders predict onset of psychotic disorder or persistent attenuated psychotic symptoms in patients at high clinical risk?

Date:

2020-06-01

\section{Citation:}

Hadar, H., Zhang, H., Phillips, L. J., Amminger, G. P., Berger, G. E., Chen, E. Y. H., de Haan, L., Hartmann, J. A., Hickie, I. B., Lavoie, S., Markulev, C., McGorry, P. D., Mossaheb, N., Nieman, D. H., Nordentoft, M., Riecher-Rossler, A., Schafer, M. R., Schloegelhofer, M., Smesny, S. ,... Nelson, B. (2020). Do schizotypal or borderline personality disorders predict onset of psychotic disorder or persistent attenuated psychotic symptoms in patients at high clinical risk?. SCHIZOPHRENIA RESEARCH, 220, pp.275-277. https://doi.org/10.1016/ j.schres.2020.03.065.

Persistent Link:

http://hdl.handle.net/11343/250293 